Bosak, Keith, and Kathleen Schroeder. 2005. "Using geographic information systems (GIS) for gender and development." Development in Practice 15, no. 2: 231-237.

Routledge, Taylor \& Francis ISSN: 09614524

Keywords: GEOGRAPHIC information systems | GENDER studies

\title{
Using Geographic Information Systems (GIS) for gender and development
}

Keith Bosak and Kathleen Schroeder

\begin{abstract}
:
This paper focuses on the use of geographic information system (GIS) in gender research and argues for the possibility that the tool has inherent biases with regard to data collection and representation.
\end{abstract}

\section{Introduction}

Given the mandate by large agencies to address gender in all development projects and the 'Mainstreaming of Gender' at the World Bank, we can expect that new opportunities will emerge for gender research. Geographic Information Systems (GIS) could play a growing part in this agenda. Though most use of GIS is still oriented towards land-use studies, geographers and others are beginning to use GIS to examine complex human variables including gender. Development practitioners should be aware both of the potential usefulness of GIS for gender analysis and of recent critiques of this powerful tool.

The debate between those informed by traditional scientific methods based on the idea of objectivity and those looking to understand the complexity of human variables who accept subjectivity as part of the research agenda provides a foundation for exploring the use of GIS for gender and development. For example, almost ten years ago a group of feminist geographers asked the question: 'Should women count?' (Mattingly and Falconer-Al-Hindi 1995), in order to address the possibility of doing feminist research using quantitative techniques. The authors argued that feminists should use whatever tools necessary to build understanding of the gendered nature of our society without forgetting that those tools themselves also have gendered biases. Powerful new technologies such as GIS have prompted people to consider their useful application to a host of complex problems, including issues of gender. We can expect that GIS will have increasing applications in gender and development.

We argue in this paper that GIS has some inherent biases with regard to data collection and representation, and that if these biases are not addressed at a theoretical or epistemological level, the use of GIS for addressing gender issues in development will be severely limited. As a primary example we use our own research project funded by the 
Consultative Group on International Agricultural Research (CGIAR) through the Participatory Research on Gender Analysis (PRGA) initiative. The goal of this project was to use a combination of GIS and qualitative methods (interviews and archival analysis) in order to determine 'hot spots' of female poverty in Nepal, Bolivia, and Malawi.

After providing a suitable definition for GIS, we examine the growing literature that provides a critique of GIS, including the new feminist discourse on the field. In particular we question the use of widely available data sources as inputs into a gendered GIS and the ability of GIS to represent women's issues. We conclude with a discussion of the possibilities for GIS to overcome these biases and be redefined as a feminist method. Within a development context, such a redefinition could provide agencies with a powerful tool with which to represent women's experiences to decision makers.

\section{Defining GIS for gender and development applications}

The acronym GIS is widely used without much attention to how it is defined. This has proved particularly problematic as we struggle to examine GIS and its usefulness for gendered research in a critical fashion. Probably the most practical way to define GIS is by its function. Most of the time, when someone refers to a GIS, s/he is referring to a database in which the variables have a locational attribute, allowing them to be mapped and manipulated. An example of this would be demographic data collected at the village level, with the location $(x, y)$ of the village given as an attribute. Such data may be overlaid on land-use data to show the relationship between economics and environmental change. This database can then be used to model phenomena in such a way as to provide what many simply accept as an accurate representation of reality.

The visual representations of these models are often used in support of decisions made by policy makers and researchers and are themselves referred to as a GIS. Recently, GIS has also been used to refer to a field. What we mean by this is that people who 'do GIS work' have created a specific niche for themselves. Someone working for a GIS firm may work on many different projects from modeling land-use change to locating pockets of impoverished women farmers. Lo and Yeung (2002) define GIS as a computer-based system used to manipulate and represent spatially referenced data. We also acknowledge that GIS is practiced as a profession that uses this system as its primary tool for the production of spatial information. As such, GIS is practiced within a social context and is subject to the motivations of its practitioners. It is this part of the definition of GIS which provides an arena for addressing issues of gender and development.

\section{Applications of GIS in development}

Although GIS can be expensive and require imported technology, Lakshman Yapa (1991) has argued that it is possible to produce systems that are compatible with the principles of appropriate technology. Agencies such as those associated with the CGIAR centres often have fairly large and well-staffed GIS laboratories. In Malawi, a powerful partnership among governmental and non-governmental agencies is extending the limits of GIS in 
terms of poverty mapping. The Malawi 2002 Atlas of Social Statistics provides extensive coverage of a wide range of poverty indicators (National Statistical Office of Malawi 2002). In Nepal, GIS has been used extensively for development applications. The International Centre for Integrated Mountain Development (ICIMOD) for example, has a well-staffed GIS department that is doing sophisticated spatial analysis. In a recent study, ICIMOD used GIS to map indicators of development at the district level. This project used a breadth of variables such as literacy rates, population density, agricultural credit, and slope steepness to construct a development index for each district. The districts were then ranked in order of most to least developed. The product was published in a book form for use by development practitioners and policy makers in Nepal (ICIMOD 1997).

With regard to studies of gender and development, the serious use of geographic information technologies is in its infancy. However, as large development agencies continue to 'mainstream' gender, the use of GIS is inevitable. For example, CGIAR also has a system-wide initiative that focuses on gender.1 This initiative is exploring the functionality of GIS to help answer questions about gender and development. CGIAR has funded a pilot project to map the poorest of poor women in three impoverished rural regions (in Nepal, Malawi, and Bolivia) at the smallest possible unit of analysis (in most cases the third administrative unit).

This is just one step towards developing sophisticated gendered databases to help development agencies better target recipient populations.

\section{Critical GIS and feminist geography}

In 1995, John Pickles provided a much-needed critique of GIS as objective science by arguing that it was subject to the inherent biases of its users. These biases manifest themselves in the collection, manipulation, and representation of data. The volume he edited (Pickles 1995) argued that GIS is affected by the social, political, and economic structures of society. The idea that GIS is deeply embedded in our social and political framework and therefore affects and is affected by political, economic, and social motivations, implies that even though GIS is treated as an unbiased view of reality, it is indeed subject to the emotions and ambitions of its practitioners. Pickles was one of the first to criticize GIS users for not acknowledging and addressing the wider social contexts within which GIS operates. These social contexts affect what types of data are collected and how they are collected as well as how the data are manipulated and represented. In this way, GIS has the potential to empower or disenfranchise and it can simultaneously do both (Harris and Weiner 1998; Janelle and Hodge 2000).

International development agencies have embraced GIS much as the business world has done. GIS allows agencies to manage large and complex spatial data sets and analyze these data in sophisticated ways. At the same time, many of these same development agencies are increasing their attention to gender issues in their development projects. It is natural that these two agendas should collide and provide an opportunity for feminist geographic research. 
Feminist geographers have recently turned their attention towards issues of gender and GIS. Mei-Po Kwan's article in the Annals of the Association of American Geographers (2002) argues that GIS can enhance feminist research by providing ways to understand the gendered experiences of individuals. She proposes that feminist GIS is not about discovering universal truths but about depicting aspects of everyday life. She reminds us that our vision is always partial and that objectification can occur through everyday language as well as through advanced technologies such as GIS. Kwan challenges geographers to move beyond dualistic divisions between quantitative and qualitative research to build more complete understandings of the real world. Kwan's article officially launches a debate that had been brewing for several years in back corners of the field, and the fusion of GIS and feminist theory should be a productive area of research for years to come.

Further evidence that geographers are beginning to examine the intersection of feminist theory and critical GIS is a special issue of Gender, Place and Culture published in 2002. In a summary statement in that issue, Susan Hanson (2002) comments that the ability GIS allows to easily change scale of analysis could make it a powerful tool for feminists. In particular, feminists whose work has pointed out the generalizations that provide a homogenized view of women's experiences could benefit from the functionality of GIS.

In the international realm, GIS has been adopted by development planners and is an increasingly important component in the decision-making processes of large international agencies. However, there is virtually no critical examination of this technology within the development literature. Much as development experts critically examine socio-economic data gathered and published by the UN, the World Bank, and other organisations, the same care needs to be applied with what is produced by GIS.

\section{Questions relating to data sources}

Data collection is extremely costly and time consuming, and the ability to share data has benefits to all concerned. Commercially produced data sets, of varying quality, are becoming increasingly available. Development planners hope to make the best use of the expensive data that they have gathered or purchased from other institutions and are looking for better systems to organize their data. Geographic information systems have proved themselves to be an excellent tool for the storage, management, manipulation, and interpretation of these data sets.

The most important obstacle to developing a gender-sensitive GIS has been the lack of gender-specific data sources. For example, some of the highest quality data available measure poverty indicators at the household level, even though research conducted decades ago argued convincingly that household-level data frequently mask inequality within households (Sen 1981). Finding gender-specific demographic data can be extremely difficult, particularly at the third administrative level. Although most countries have census data in some form, the extent of gender-specific data tends to be limited to sex ratios, total fertility rates, and female literacy rates. Variation by country of the quality 
and quantity of data is enormous. For example, until very recently Malawi had little reliable census data below the national level. The Bolivian Census only collects data on sex ratios, female literacy, and educational achievement, while Nepal has some of the richest gender-specific data. The richness of data available for Nepal probably reflects its position as a key location for development projects. In addition, most of the data for Nepal are already in a digital format that can be used in GIS applications. The availability of data can drive research agendas which in turn can mean that some of the poorest and least enfranchised populations remain ignored.

Far more and better quality data are available through Macro International, the institution that organizes and conducts Demographic and Health Surveys (DHS) worldwide. DHS data have been widely used and a growing literature addresses their background and inherent problems (Gould 1995; Cleland 1996). These surveys provide excellent information on a wide variety of topics relating to health and well-being, which address women's issues specifically. Since 1998, Macro International has been using Global Positioning Systems (GPS) technology to attach geographic coordinates to their survey locations in some countries. However, the locations of villages are given random errors in order to protect the identity of the women. Therefore, these point data are not useful for geographers wanting to use the surveys to represent women's status or conditions within an area.

Data collection is perhaps the point where most inadvertent gender bias is introduced into GIS. Often the data collected are either simply not tailored towards women's issues, or the data such as that available from DHS do address women's issues directly but not in a manner that can be used in GIS. The next section explores how to represent women and development issues in a GIS, given the limitations of available data.

\section{GIS and the representation of women}

Sara McLafferty (2002) provides an exciting example of how GIS helped empower (and fund) research on breast cancer in Long Island, New York. She is also careful to point out that the success of the project she describes would have been unlikely had it not been located in a wealthy and politically well-connected community. In addition, she explains that as the grassroots initiative evolved into a multi-million dollar GIS, women lost much of the control of the project. If this can happen to well-educated white women in the USA, what are the chances that impoverished women in the developing world will exercise control over GIS technology designed for their 'betterment'?

Kwan (2002) suggests four strategies that may help to better represent women with GIS. The first is to complement secondary data with other contextual information. For example, data for Nepal show that women in the Eastern Terai region bordering the Indian state of Bihar have the lowest average body mass of any region in the country. The Terai is Nepal's most agriculturally productive region, yet women living there are undernourished. In fact, much of the food grown in the Terai is exported to Bihar, a state ravaged by poverty. This contextual information provides a starting point for further enquiry into women's nutrition in the Eastern Terai. 
Second, Kwan suggests collecting primary data from individuals. These data can be quantitative, qualitative or both. Data-collection instruments can be developed which specifically address women's issues. Our project used available data from Census (quantitative secondary data) and other sources combined with archival research and interviews with local development practitioners (qualitative primary data) in order to determine 'hot spots' of female poverty. Next, data manipulation and analysis should be carried out using or developing appropriate procedures rather than relying on inappropriate but easily accessible procedures already being used in GIS.

The final strategy is reflexivity. Reflexivity is a basic part of feminist research which requires transparency in motivation and self-reflection. Both provide insight into the standpoint of the researcher as well as the dynamic that occurs between researcher and participant. The researcher must reflect on his or her own actions, thoughts, and feelings in order to gain perspective on how s/he affects and is affected by participants. Kwan argues that reflexivity in GIS should be practiced at every stage, with particular attention paid to the data-collection and representation or visualization stages. She also recommends using multiple methods in order to gain a contextualised understanding of the problem. An example of this is the use of demographic data for poverty analysis combined with interview data from the same area. Both methods provide a partial perspective of the same issue. Following Kwan's strategies provides for some interesting possibilities for creating a GIS for addressing gender and development issues.

\section{Possibilities}

This section discusses the possibilities for creating a GIS that is capable of addressing issues of gender and development. We argue that the intersection of GIS with feminist thought provides the best path for exploring such possibilities. Feminist epistemology includes ideas of situated knowledges, representation, and reflexivity. Many of these ideas are represented through qualitative research. When these feminist principles are applied to GIS, a redefinition occurs and new possibilities arise.

Some feminist postmodernists see GIS as the embodiment of the discourse of science. There is a distinct separation of subject from object, the belief in a single truth that can be uncovered using rational thought and scientific methodologies. But theoreticians such as Donna Haraway (1991) have a modified view of objectivity that could use GIS in order to combine partial perspectives into a more objective representation of reality. We argue that this because GIS is made up of a series of data layers. Each layer could represent a partial perspective. The layering of many localized partial perspectives could possibly offer a more complete form of knowledge. The combination of secondary data with primary data, which may be qualitative (as shown in the example from the Terai of Nepal), provides several partial perspectives of a single issue.

Positivist epistemology is most closely associated with modern scientific 
methodologies such as GIS. Positivism states that human behavior is governed by laws similar to nature, and attempts to apply the scientific method to social science. Hypothesis formulation and verification is an integral component of positivist science. Critics of positivism (such as feminists) see this philosophy/methodology as fundamentally flawed. One criticism is that there is no foundation for certain knowledge and all observations are simply theory-impregnated interpretations of facts. As such, feminist researchers often use qualitative research in order to reveal the subjective side of scientific enquiry. Qualitative research, as opposed to quantitative research, uses data that cannot be measured with numbers. Given this, it is a challenge not to define qualitative research but how to define its theory.

Qualitative researchers see the world as socially constructed and therefore seek answers to questions about how social experiences are created and given meaning. Qualitative research is based upon the notion of the researcher and researched affecting one another. Transparency in motivation reflexivity devices is necessary in order to produce good qualitative research (Denzin and Lincoln 2000). Returning to our example from the Terai of Nepal, qualitative researchers would be interested to find out how women's status in society and the home affect their access to proper nutrition and healthcare.

Gender and development researchers stand to benefit in two ways by combining qualitative methodology with GIS. Specifically, multimedia GIS can help provide a level of transparency in the research process, which has been deemed an essential component of rigorous qualitative research (Baxter and Eyles 1997). Second, multimedia GIS offers qualitative researchers a powerful tool for the organization and representation of data (Rist 2000).

Multimedia GIS ties spatially referenced pictures, video (interviews, participant observation, focus groups) archival data, as well as demographic data and other attribute data with 'real world' locations (Cartwright 1997). Many of the technical issues once involved with this kind of GIS have now been resolved allowing for integration of multimedia with GIS (Dransch 2000). Including video and audio with demographic data gives researchers and decision makers a perspective not from space but from the level of the research participant. In other words, multimedia and participatory GIS can help the user realize that the phenomenon under study occurs at some real, specific, and complex location. Seeing images of real women telling their own stories can be a more effective tool for development practitioners than are color-coded maps and regression models. Giving women a space to use their voices could be far more empowering than filling out a survey.

\section{Conclusions}

In the international development sphere, GIS is now commonly used for spatial decision making. Many large development institutes have invested heavily in GIS technology and are currently conducting development research with the aid of this powerful tool. Those of us concerned with gender and development issues have much to gain from the use of 
GIS, particularly if we are aware of its ability to both empower and disenfranchise women and the poor.

Informing our geographic information systems with feminist theory could create a gendered GIS to help the impoverished. This process starts with the research questions we ask, moves into the data we collect, and is finalized in the graphic representations we produce. GIS has characteristics inherent in its structure that make it possible to produce gendered knowledge that can help women. Our job is to be cognizant of these characteristics, inform ourselves with feminist theory, and push the boundaries of what is called GIS.

\section{Acknowledgements}

This project was funded by the CGIAR Program on Participatory Research and Gender Analysis. The authors would like to thank Jacqueline Ashby for her support of this work.

\section{Note}

1. See www.prgaprogram.org for more information.

\section{References}

Baxter, J. and J. Eyles (1997) 'Evaluating qualitative research in social geography: establishing 'rigor' in interview analysis', Transactions of the Institute of British Geographers, NS 22:505 - 525.

Cartwright, W. (1997) 'New media and their application to the production of map products', Computers and Geosciences 23(4):447-456.

Cleland, J. (1996) 'Demographic data collection in Less Developed Countries 19461996', Populations Studies 50(3):433-450.

Denzin, N. and Y. Lincoln (2000) 'Introduction: the discipline and practice of qualitative research', in N. Denzin and Y. Lincoln (eds.) Handbook of Qualitative Research, Thousand Oaks, CA: Sage.

Dransch, D. (2000) 'The use of different media in visualizing spatial data', Computers and Geosciences 26(1):5 - 9 .

Gould, W. T. S. (1995) 'Ideology and data analysis in African population policies', Applied Geography 15(3):203-218.

Hanson, S. (2002) 'Connections', Gender, Place and Culture 9(3):301 - 303.

Haraway, D. (1991) Simians, Cyborgs and Women: The Reinvention of Nature, London: Free Association. 
Harris, T. and D. Weiner (1998) 'Empowerment, marginalization and "community-integrated" GIS', Cartography and Geographic Information Systems 25(2):67 -76 .

International Centre for Integrated Mountain Development (ICIMOD) (1997) Districts of Nepal Indicators of Development, Kathmandu: ICIMOD.

Janelle, D. G. and D. Hodge (eds.) (2000) Information, Place and Cyberspace, New York, NY: Springer.

Kwan, M. (2002) 'Feminist visualization: re-envisioning GIS as a method in feminist geographic research', Annals of the Association of American Geographers 92(4):645661.

Lo, C. P. and A. K. W. Yeung (2002) Concepts and Techniques of Geographic Information Systems, Upper Saddle River, NJ: Prentice Hall.

Mattingly, D. and K. Falconer-Al-Hindi (1995) 'Should women count? A context for the debate', The Professional Geographer 47(4):427- 435.

McLafferty, S. L. (2002) 'Mapping women's worlds: knowledge, power and the bounds of GIS', Gender, Place and Culture 9(3):263 - 269.

National Statistical Office of Malawi (2002) Malawi 2002 Atlas of Spatial Statistics, available at www.nso.malawi.net (accessed 10 July 2004).

Pickles, J. (ed.) (1995) Ground Truth: The Social Implications of GIS, New York, NY: Guilford Press.

Rist, R. (2000) 'Influencing the policy process with qualitative research', in N. Denzin and Y. Lincoln (eds.) Handbook of Qualitative Research, Thousand Oaks, CA: Sage.

Sen, A. (1981) Poverty and Famines: An Essay on Entitlements and Deprivations, Oxford: Clarendon Press.

Yapa, L. (1991) 'Is GIS appropriate technology?', International Journal of Geographic Information Systems 5(1):41 - 58. 